\title{
Adaptive Rendering based on Weighted Local Regression
}

\author{
Bochang Moon ${ }^{1}$, Nathan Carr², Sung-Eui Yoon ${ }^{1}$ \\ $\mathrm{KAIST}^{1}$, Adobe $^{2}$
}

\section{SUPPLEMENTARY REPORT}

Contents

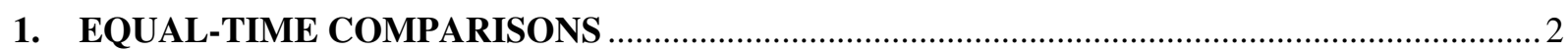

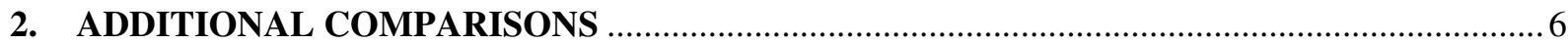

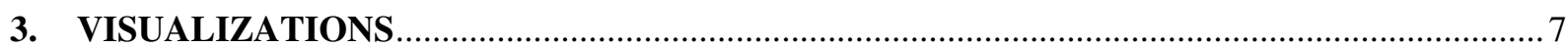

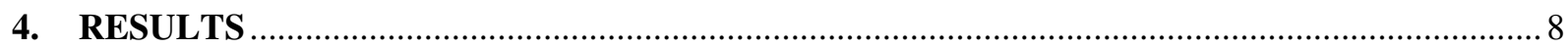

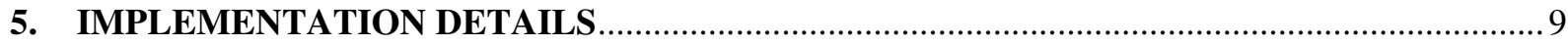




\section{EQUAL-TIME COMPARISONS}

We compare our method with LD (low discrepancy sampling), non-local means (NLM) sampling [Roussselle et al. 2012], cross bilateral filtering based on Stein's unbiased risk estimator (SURE) descried in [Li et al. 2012], and BM3D based wavelet denoising (BM3D) [Kalantari and Sen 2013]. As a quantitative measure for comparisons we use the relative MSE (rMSE) defined in [Rousselle et al. 2011].

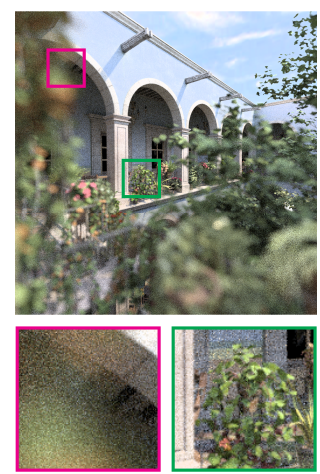

(a) LD $128 \mathrm{spp}$ (665 s) rMSE 0.06288

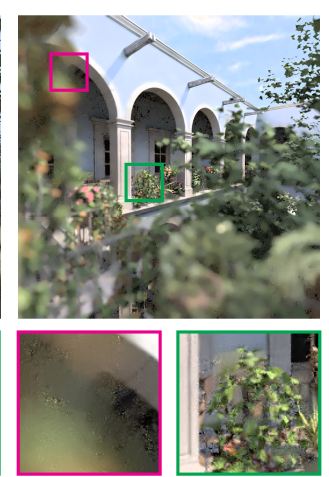

(b) NLM $115 \mathrm{spp}$ (665 s) rMSE 0.01242

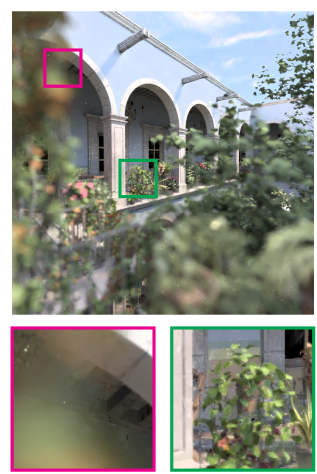

(c) SURE $113 \mathrm{spp}$ (665 s) rMSE 0.01521
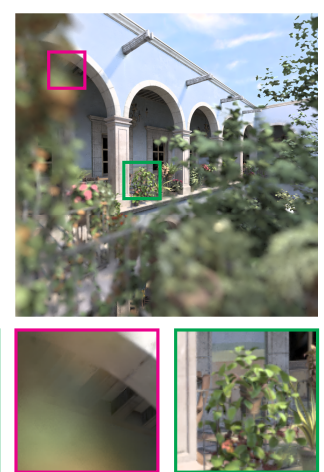

(d) Ours $115 \mathrm{spp}$ (660 s) rMSE 0.00448
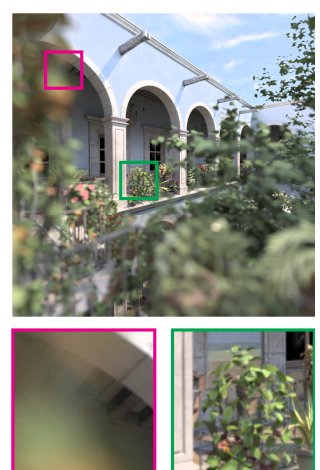

(e) Reference $16 \mathrm{~K} \mathrm{spp}$

Fig. 1. Same-time comparisons in San Miguel scene.

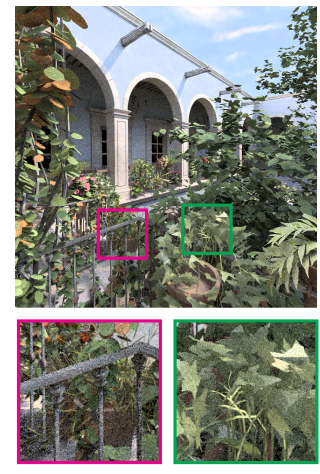

(a) LD $128 \mathrm{spp}$ (654 s) rMSE 0.04817

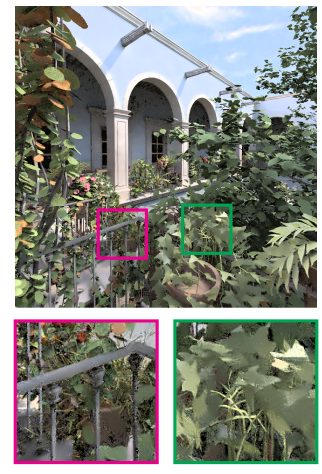

(b) NLM $115 \mathrm{spp}$ (654 s) rMSE 0.02817

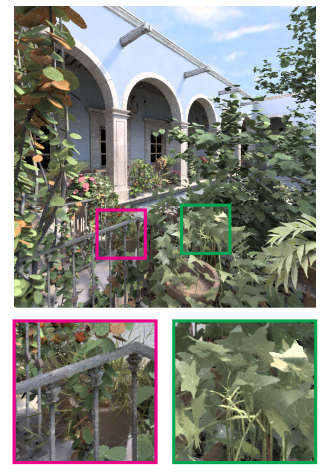

(c) SURE $114 \mathrm{spp}$ (656 s) rMSE 0.01964

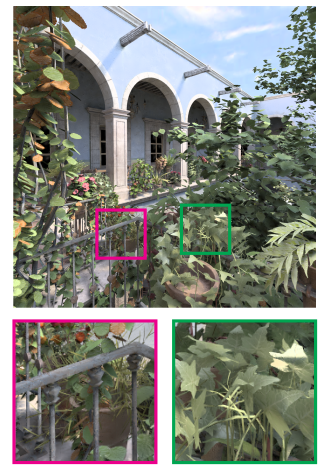

(d) Ours $109 \mathrm{spp}$ (653 s) rMSE 0.00917

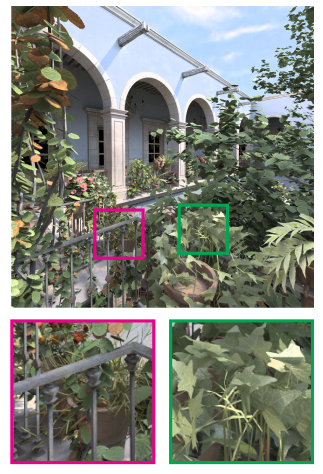

(e) Reference $16 \mathrm{~K}$ spp

Fig. 2. Same-time comparisons in San Miguel scene (without depth-of-field). 


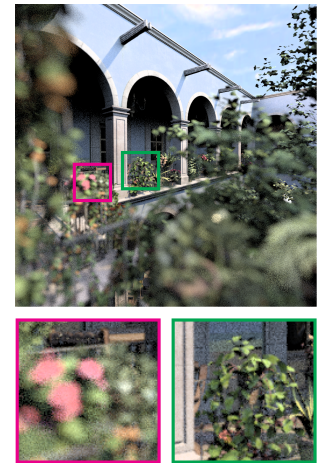

(a) LD $24 \mathrm{spp}$ (750 s) rMSE 0.02852

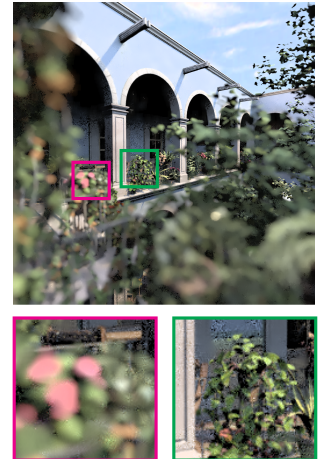

(b) NLM $20 \mathrm{spp}$ (539 s) rMSE 0.00944

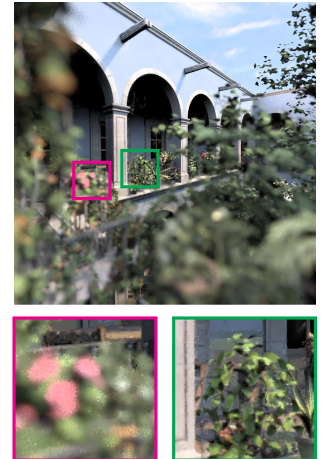

(c) SURE $16 \mathrm{spp}$ (546 s) rMSE 0.02207

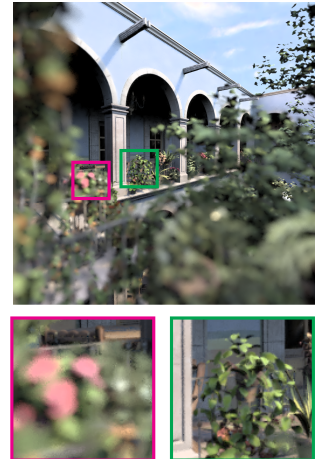

(d) Ours $16 \mathrm{spp}$

(536 s) rMSE 0.00728

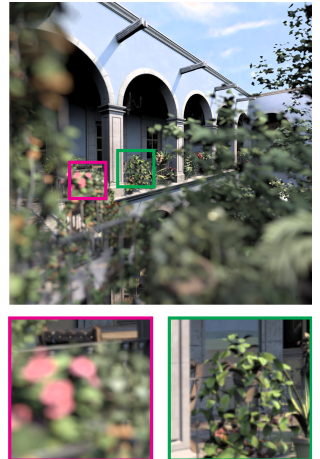

(e) Reference $4 \mathrm{~K} \mathrm{spp}$

Fig. 3. Comparisons in San Miguel. The indirect illumination is disabled for this test.
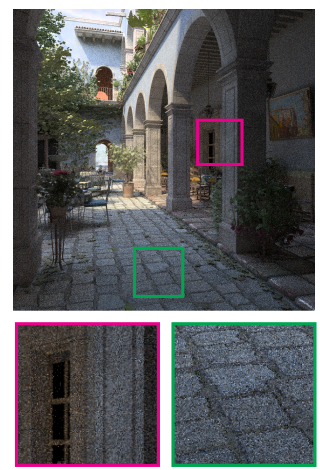

(a) LD $128 \mathrm{spp}$ (1346 s) rMSE 0.06273
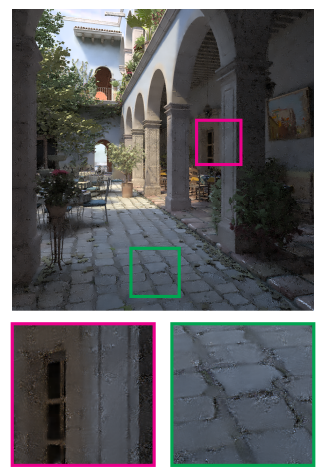

(b) NLM $120 \mathrm{spp}$ (1242 s) rMSE 0.02030
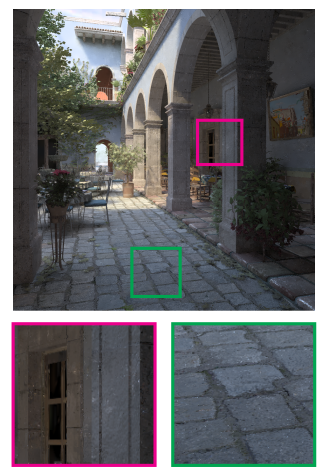

(c) SURE $120 \mathrm{spp}$ (1234 s) rMSE 0.01154
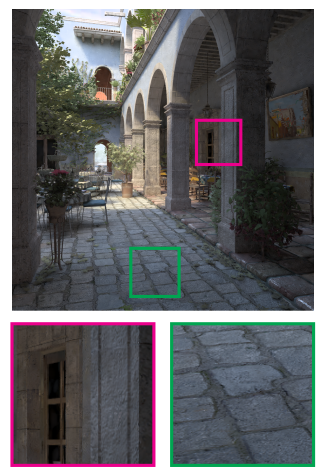

(d) Ours $117 \mathrm{spp}$ (1231 s) rMSE 0.00695

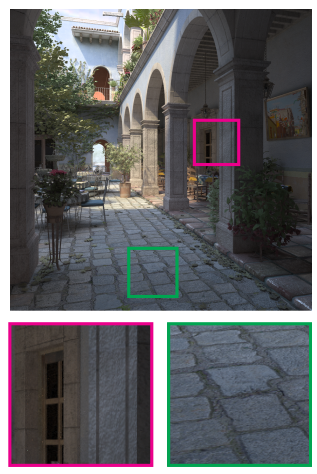

(e) Reference $16 \mathrm{~K} \mathrm{spp}$

Fig. 4. Comparisons with another camera view. The San Miguel scene are rendered by path tracing.
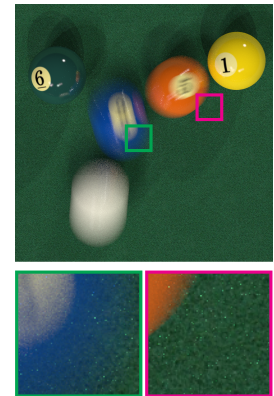

(a) LD $53 \mathrm{spp}$ (93 s)

rMSE 0.01684
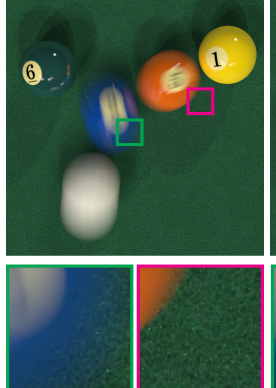

(b) NLM $41 \mathrm{spp}$ (91 s) rMSE 0.00404
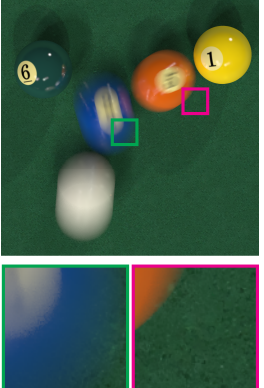

(c) SURE $35 \mathrm{spp}$ (92 s) rMSE 0.00130
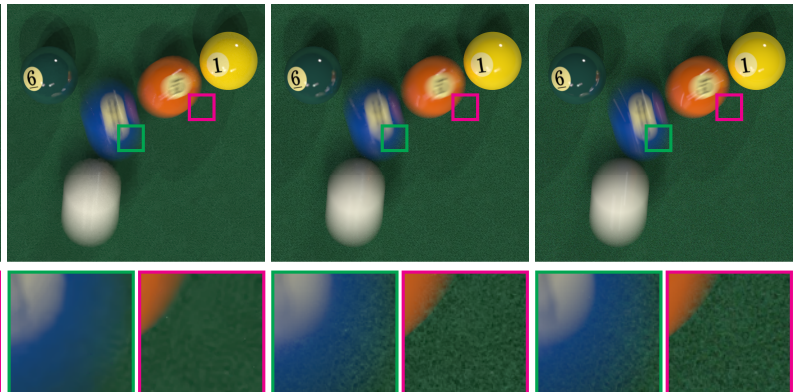

(d) BM3D

$32 \mathrm{spp}$ rMSE 0.00349

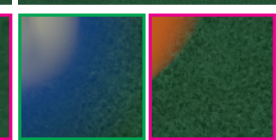

(e) Ours $32 \mathrm{spp}$ (91 s) rMSE 0.00044

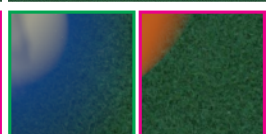

(f) Reference $64 \mathrm{~K} \mathrm{spp}$

Fig. 5. Same-time comparisons in pool scene. 


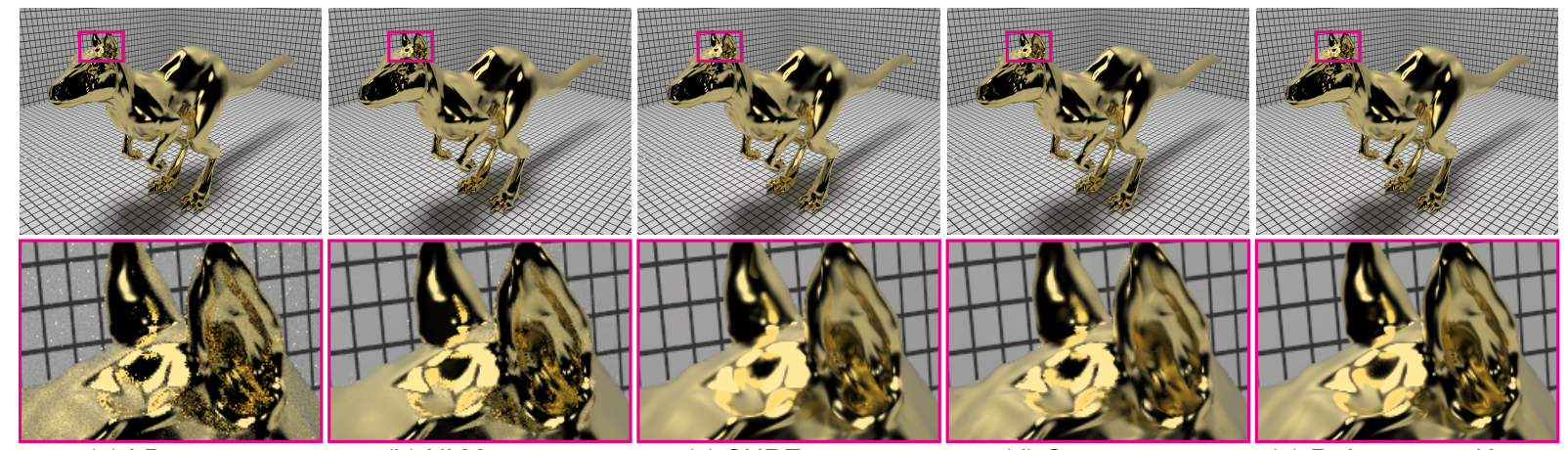

(a) LD $32 \mathrm{spp}$

(79 s) rMSE 0.02384

(b) NLM $24 \mathrm{spp}$

(79 s) rMSE 0.00417 (c) SURE $22 \mathrm{spp}$

(79 s) rMSE 0.00576 (d) Ours $16 \mathrm{spp}$

(78 s) rMSE 0.00206

Fig. 6. Equal-time comparisons in killeroo-gold scene.

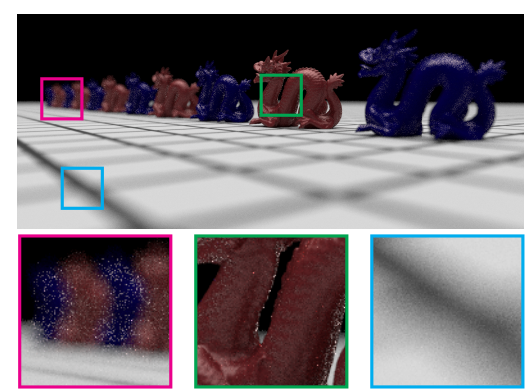

(a) LD $39 \mathrm{spp}(66 \mathrm{~s})$ rMSE 0.01350

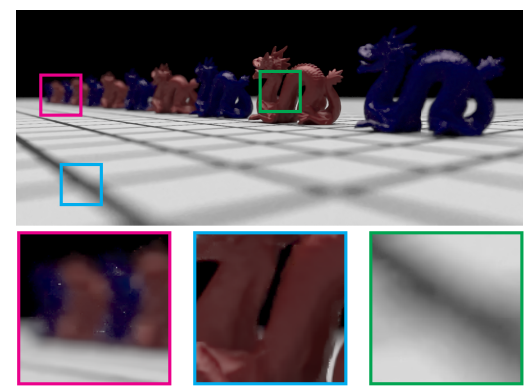

(d) BM3D $16 \mathrm{spp}$ rMSE 0.00443

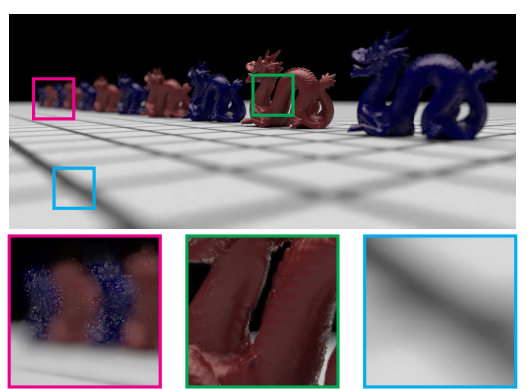

(b) NLM 17 spp (66 s) rMSE 0.00447

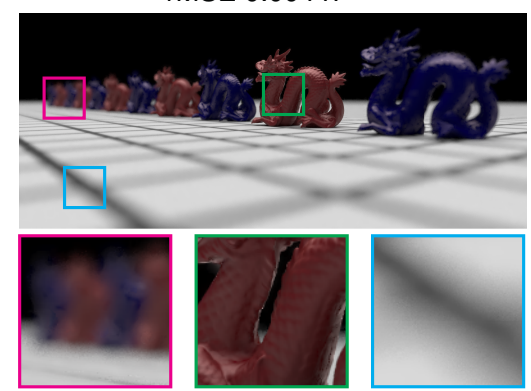

(e) Ours $16 \mathrm{spp}$ (66 s) rMSE 0.00316

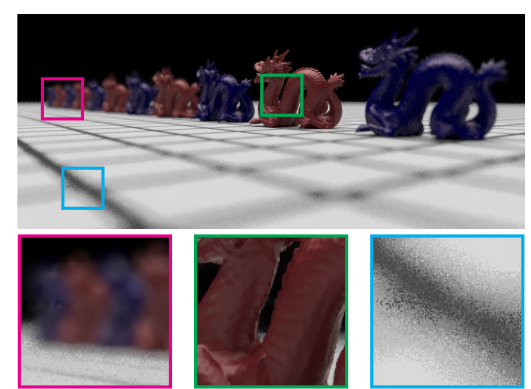

(c) SURE $15 \mathrm{spp}$ (66 s) rMSE 0.00678

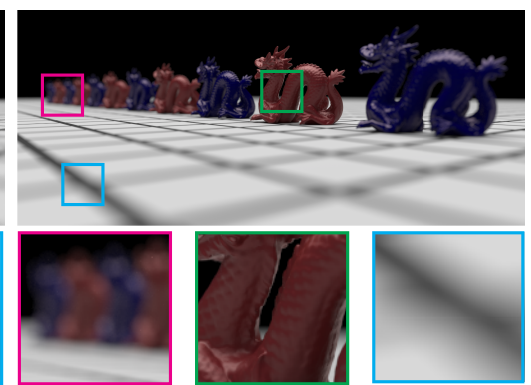

(f) Reference $64 \mathrm{~K}$

Fig. 7. Equal-time comparisons in dof-dragons scene. 

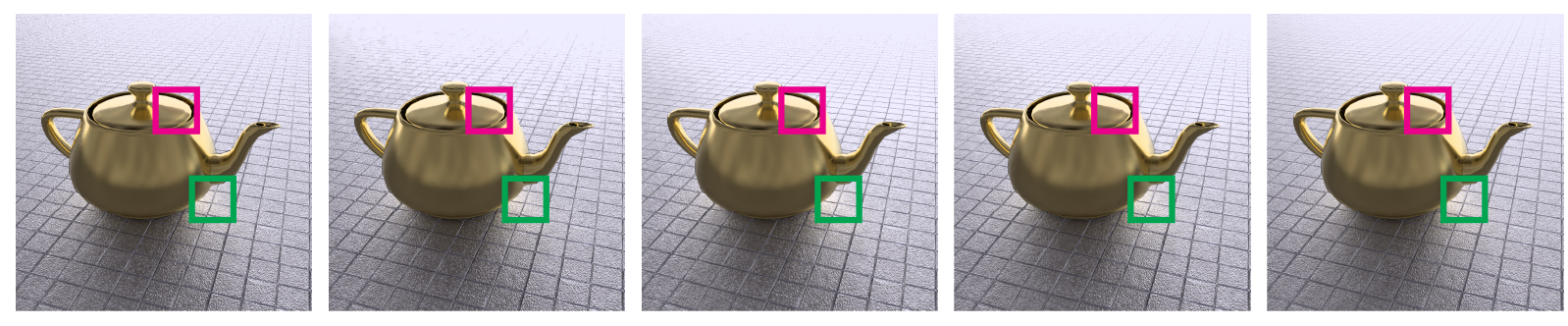

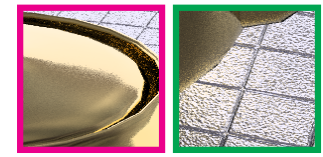

(a) LD $80 \mathrm{spp}$

(140 s) rMSE 0.03898

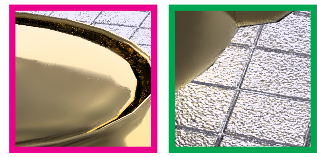

(b) NLM $76 \mathrm{spp}$ (138 s) rMSE 0.03371

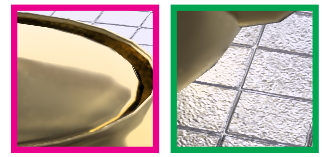

(c) SURE $67 \mathrm{spp}$ (138 s) rMSE 0.03755

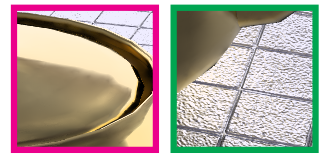

(d) Ours $62 \mathrm{spp}$ (138 s) rMSE 0.02248

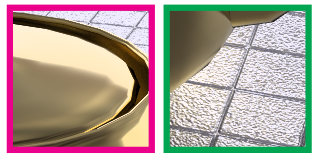

(e) Reference $16 \mathrm{~K} \mathrm{spp}$

Fig. 8. Equal-time comparisons in teapot scene.

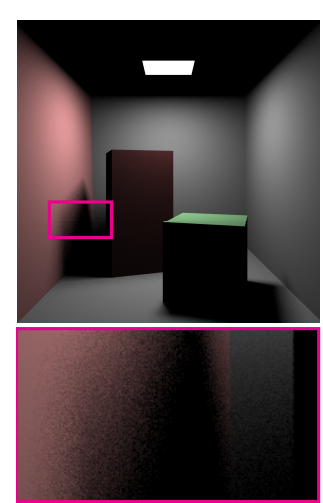

(a) LD $17 \mathrm{spp}$ (10 s)

rMSE 0.00070

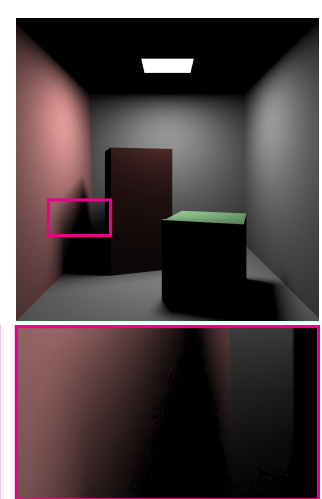

(b) NLM $9 \mathrm{spp}$ (10 s)

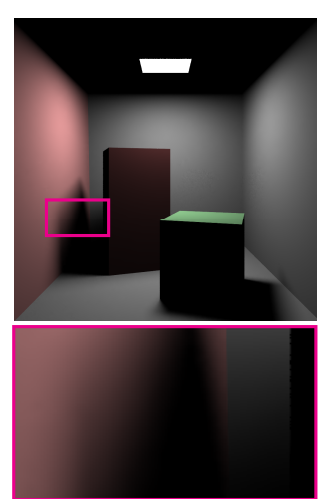

(c) SURE $7 \mathrm{spp}$ (10 s) rMSE 0.00017

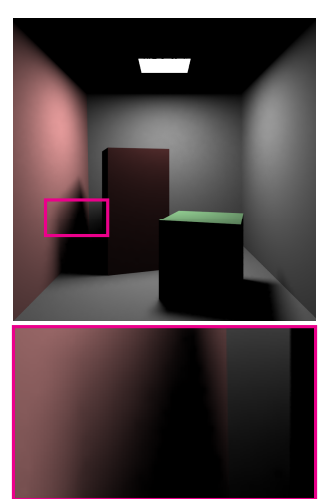

(d) Ours $4 \mathrm{spp}$ (10 s) rMSE 0.00008

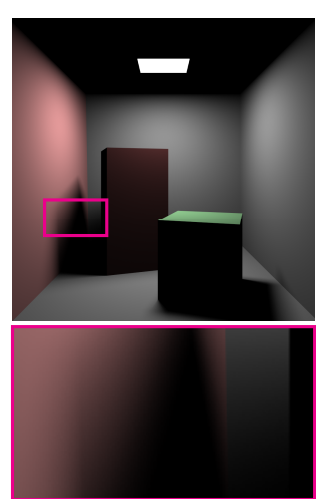

(e) Reference $4 \mathrm{~K}$

Fig. 9. Equal-time comparisons in cornell box. Our method (d) uses a very small sample count (e.g., 4), but the soft shadows are properly reconstructed. 


\section{ADDITIONAL COMPARISONS}
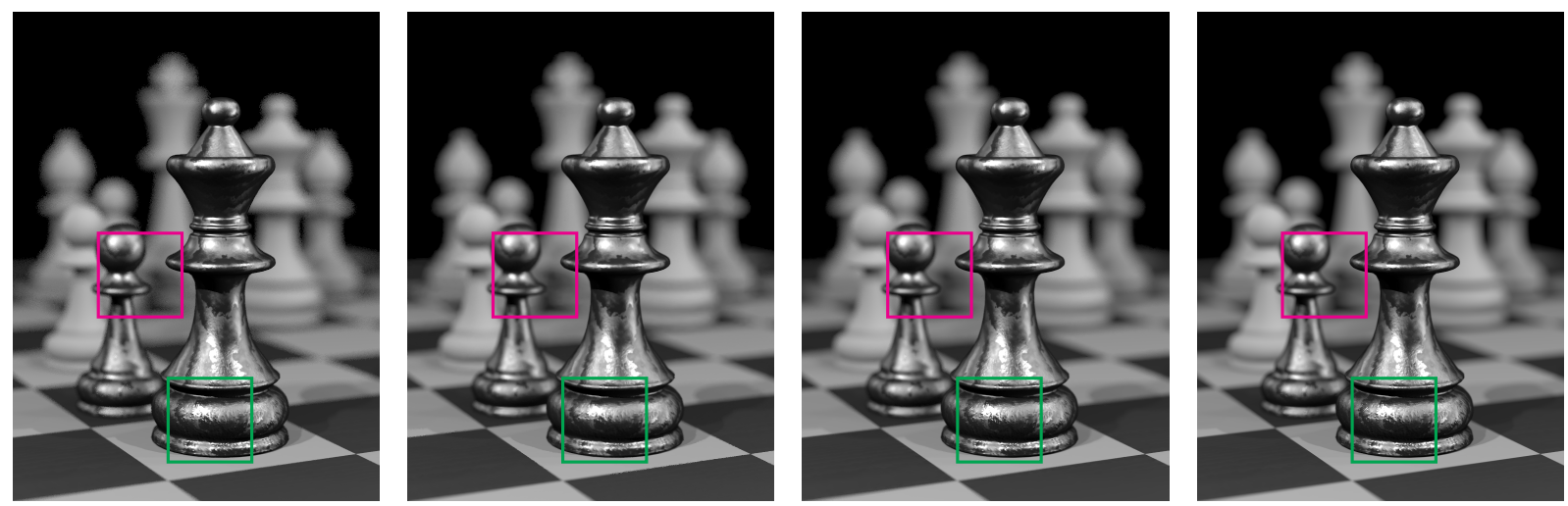

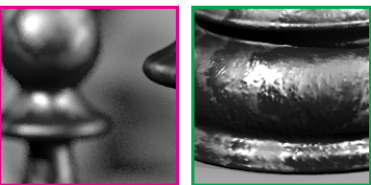

(a) LD $16 \mathrm{spp}$ rMSE 0.00122

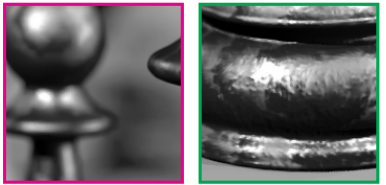

(b) BM3D $16 \mathrm{spp}$ rMSE 0.00080

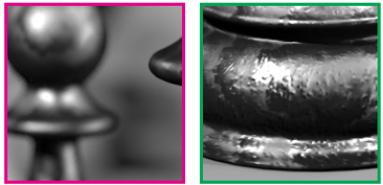

(c) Ours $16 \mathrm{spp}$

rMSE 0.00032

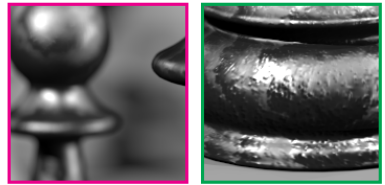

(d) Reference $4 \mathrm{~K} \mathrm{spp}$

Fig. 10. Comparisons in the chess scene. Indirect illumination is disabled for this test.
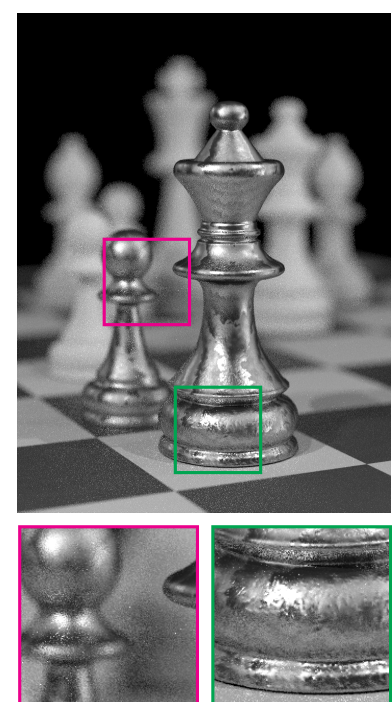

(a) LD $48 \mathrm{spp}$ rMSE 0.02035
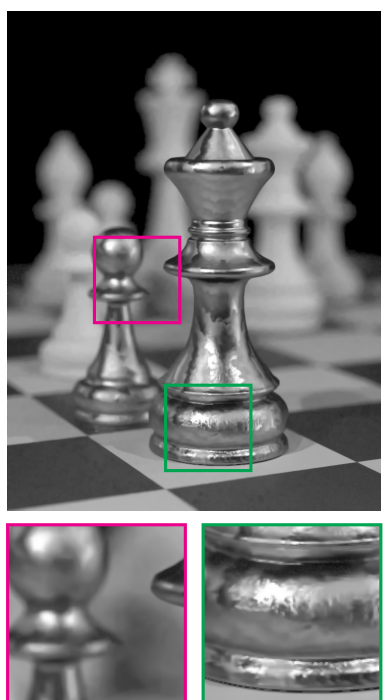

(b) BM3D $48 \mathrm{spp}$ rMSE 0.00193
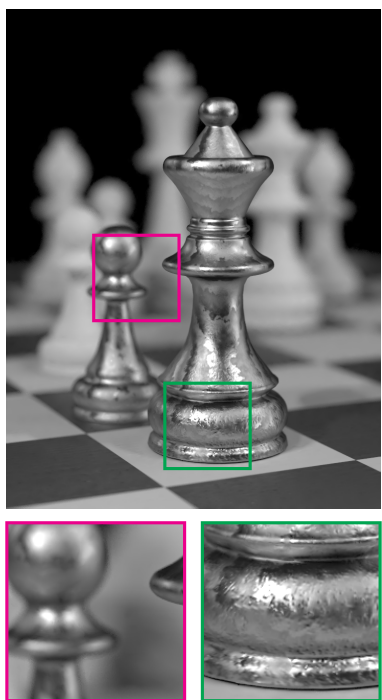

(c) Ours $48 \mathrm{spp}$ rMSE 0.00078
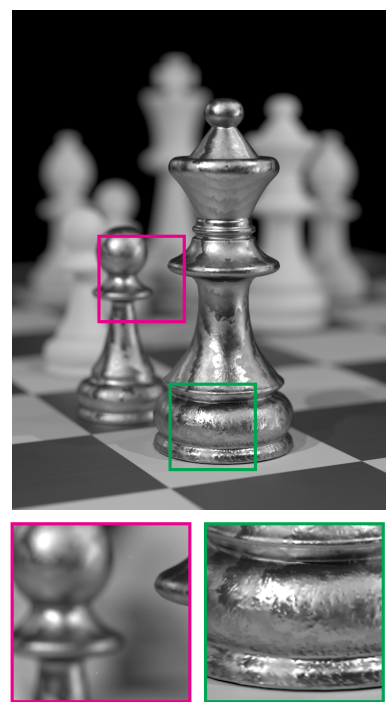

(d) Reference $16 \mathrm{~K} \mathrm{spp}$

Fig. 11. Path tracing results in the chess scene. 


\section{VISUALIZATIONS}

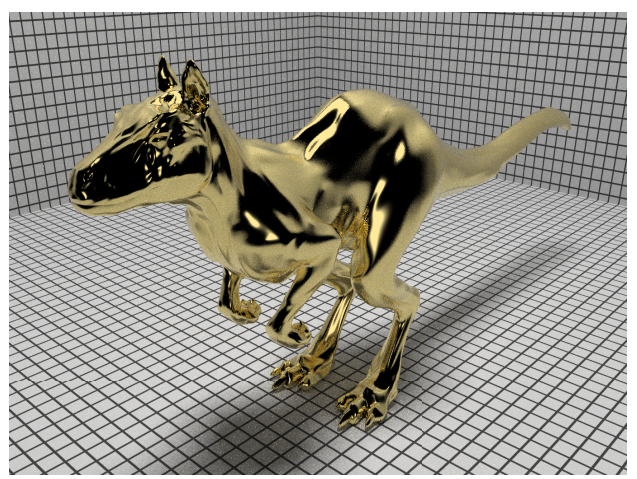

(a) Input $32 \mathrm{spp}$

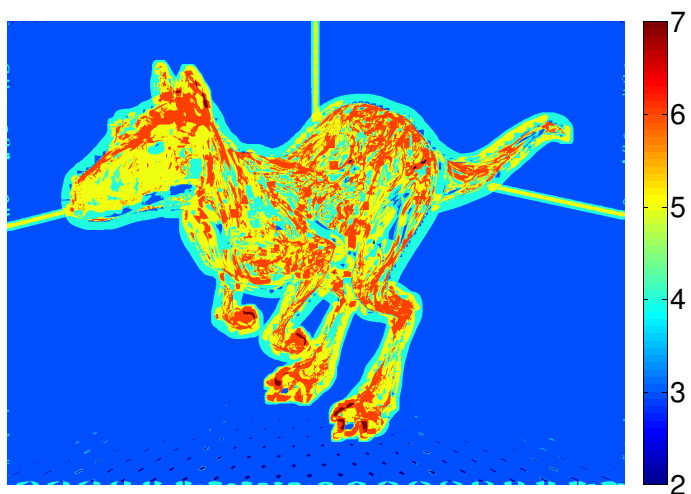

(b) local dimension by our TSVD

Fig. 12. Estimated local dimensions of the killeroo-gold scene. In the textured floor area, we have a low rank (e.g., 3) since the truncated SVD removes dependency in geometric features (e.g., textures, normals, and depth). The estimated average rank is 3.5.

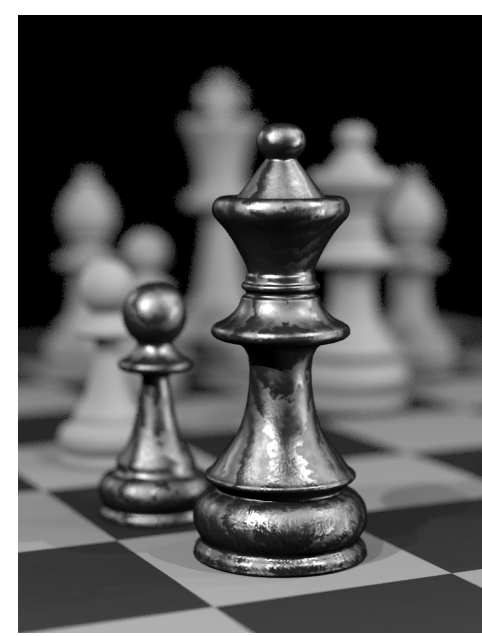

(a) Input $16 \mathrm{spp}$

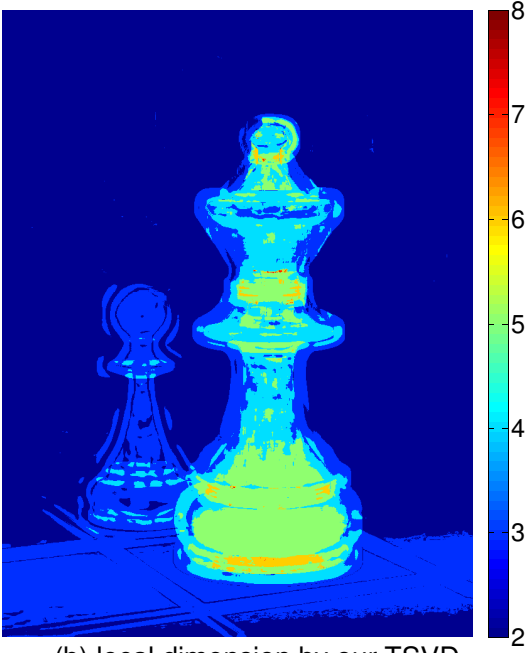

(b) local dimension by our TSVD

Fig. 13. Estimated local dimensions of the chess scene. We use uniformly generated 16 samples per pixel for the input image (a). In the computed dimension image (b), the estimated local rank on the bumped textures is larger than that of defocused regions. 


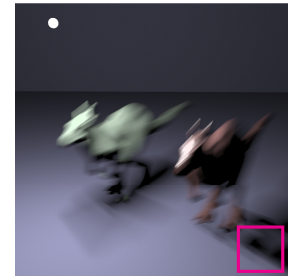

(a) Input $16 \mathrm{spp}$

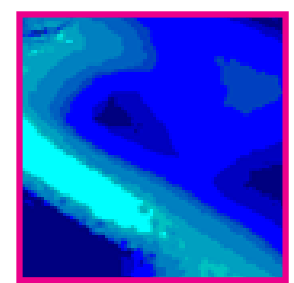

(f) Estimated standard deviation

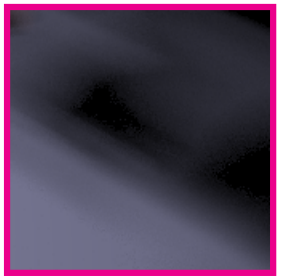

(b) Inset of (a)

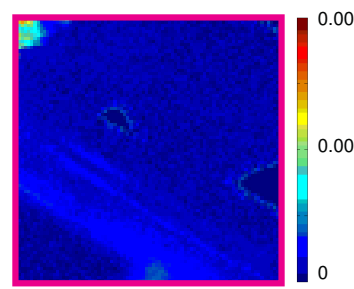

(g) Reference standard deviation

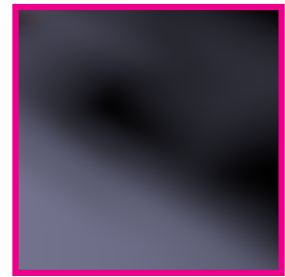

(c) Result with $\mathrm{h}=1$

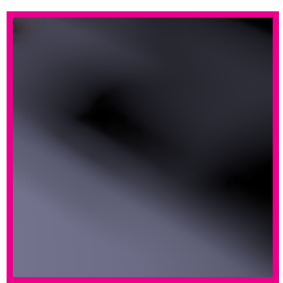

(h) Our result with estimated errors rMSE 0.00018

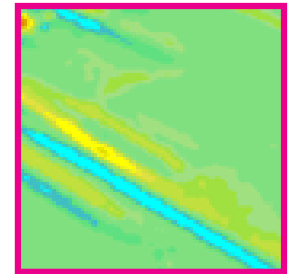

(d) Estimated bias

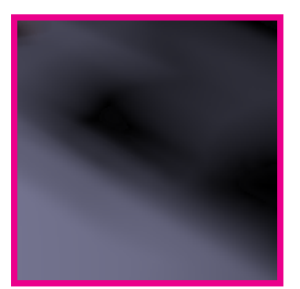

(i) Our result with reference errors rMSE 0.00009

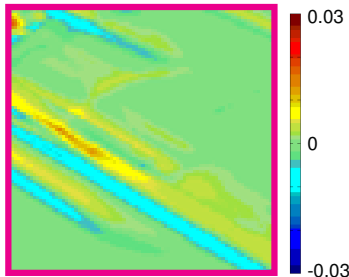

(e) Reference bias

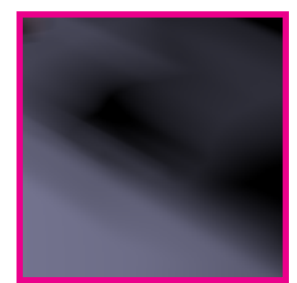

(j) Reference $4 \mathrm{~K} \mathrm{spp}$

Fig. 14. Failure case of iid assumption (i.e., independent and identically distributed random samples). Given the anim-killeroos-moving scene, we compare our estimated error ( $\mathrm{d}$ and $\mathrm{f}$ ) to reference errors (e and $\mathrm{g}$ ). To compute the reference errors, we apply our filtering with $\mathrm{h}=1$ on many input images (e.g., 500 images), each of which is computed by low discrepancy sampling for generating each input image. Our estimated variance (f) has overestimated values compared to the reference variance $(\mathrm{g})$, since we estimate variance based on the iid assumption. As a result, it leads to over-blurred artifacts (h) compared to our result based on the reference errors (i). The overestimation also leads to overshooting on the area since our adaptive sampling allocates ray budgets on pixels with high errors. However, the rMSE of our method (e.g., 0.00018) is not significantly higher than the oracle (0.00009). We have conducted this test again with random sampling instead of low discrepancy sampling. In this setting, our error (e.g., 0.00041 ) is only $20 \%$ higher compared to the perfect oracle (e.g., 0.00034).

\section{RESULTS}

Quantitative results of our supplementary video. Table I shows the relative MSE (rMSE) of all the tested methods in the supplementary video. Our method produces numerically more correct filtered images compared to all the other tested methods. Especially our method shows more than an order of magnitude improvement in terms of rMSE over SURE. Note that we extended SURE to utilize temporal coherence for a fair comparison with our method, although its original paper did not demonstrate it. Nonetheless, it is unclear how to optimize bandwidths for SURE in the temporal domain. Because of this issue, our method shows an even greater improvement over SURE when applied to animation. In our accompanied video we also show results of the San Miguel benchmark without using depth-of-field effects, to show how different methods behave. In this scene more high-frequency details exist. Our method shows visually and numerically better results over other tested methods. In this scene our method shows much higher visual quality and numerically better (e.g., more than two times) over NLM. NLM works poorly in this scene, 
mainly because this scene has a lot of high-frequency details, but NLM does not utilize geometric information.

Table II. rMSE comparisons of animated results

\begin{tabular}{|c|c|c|c|c|}
\hline killeroo-gold & frame 50 & frame 100 & frame 150 & frame 200 \\
\hline LD (55 spp) & 0.01372 & 0.01282 & 0.01300 & 0.01367 \\
\hline NLM (42 spp) & 0.00175 & 0.00161 & 0.00167 & 0.00158 \\
\hline SURE (42 spp) & 0.09220 & 0.06981 & 0.02699 & 0.03619 \\
\hline Ours (32 spp) & $\mathbf{0 . 0 0 1 4 1}$ & $\mathbf{0 . 0 0 1 2 5}$ & $\mathbf{0 . 0 0 1 2 6}$ & $\mathbf{0 . 0 0 1 2 4}$ \\
\hline
\end{tabular}

\begin{tabular}{|c|c|c|c|c|}
\hline San Miguel & frame 50 & frame 100 & frame 150 & frame 200 \\
\hline LD (143 spp) & 0.05433 & 0.05127 & 0.04894 & 0.04934 \\
\hline NLM (128 spp) & 0.00814 & 0.00845 & 0.00843 & 0.00962 \\
\hline SURE (126 spp) & 0.01228 & 0.01448 & 0.01402 & 0.01548 \\
\hline Ours (128 spp) & $\mathbf{0 . 0 0 5 2 5}$ & $\mathbf{0 . 0 0 5 4 6}$ & $\mathbf{0 . 0 0 5 2 8}$ & $\mathbf{0 . 0 0 5 5 6}$ \\
\hline
\end{tabular}

\begin{tabular}{|c|c|c|c|c|}
\hline San Miguel (without depth-of-field) & frame 50 & frame 100 & frame 150 & frame 200 \\
\hline LD (153 spp) & 0.04090 & 0.03987 & 0.03614 & 0.03702 \\
\hline NLM (136 spp) & 0.01828 & 0.01688 & 0.01626 & 0.01720 \\
\hline SURE (137 spp) & 0.01582 & 0.01485 & 0.01371 & 0.01391 \\
\hline Ours (128 spp) & $\mathbf{0 . 0 0 8 5 2}$ & $\mathbf{0 . 0 0 7 9 0}$ & $\mathbf{0 . 0 0 7 5 7}$ & $\mathbf{0 . 0 0 7 6 8}$ \\
\hline
\end{tabular}

\begin{tabular}{|c|c|c|c|c|}
\hline $\begin{array}{c}\text { San Miguel } \\
\text { (including a camera with translation) }\end{array}$ & frame 50 & frame 100 & frame 150 & frame 200 \\
\hline \hline LD (153 spp) & 0.03984 & 0.04493 & 0.03762 & 0.03650 \\
\hline NLM (136 spp) & 0.01380 & 0.01240 & 0.01198 & 0.01220 \\
\hline SURE (137 spp) & 0.01338 & 0.01129 & 0.01158 & 0.01177 \\
\hline Ours (128 spp) & $\mathbf{0 . 0 0 7 7 2}$ & $\mathbf{0 . 0 0 7 1 6}$ & $\mathbf{0 . 0 0 7 1 3}$ & $\mathbf{0 . 0 0 7 0 7}$ \\
\hline
\end{tabular}

\section{IMPLEMENTATION DETAILS}

Extension to temporal domain for SURE [Li et al. 2012]. The temporal extension of SURE was not described in their paper. Nonetheless, to conduct fairer comparisons we extended their cross bilateral filtering for utilizing the temporal coherence. Specifically, we add an additional Gaussian function to the cross bilateral filtering. Its distance function is defined based the difference on frame numbers of pixels. Given the modified formulation, neighboring pixels are defined as pixels within a filtering window $7 \times 7 \times$ 5 centered on a center pixel, as used in our method. The parameters of the Gaussian functions using pixel locations is set as a half size of the given filtering window size. Other parameters of the Gaussian functions using geometries is set as same values used for $2 \mathrm{D}$ filtering. The extended cross bilateral filtering with the chosen 3D filtering window is then applied to filter the images, each of which is generated by SURE, in the same manner to what we have done for our method. 
Parameters used for testing previous methods. For the non-local means (NLM) adaptive rendering [Rousselle et al. 2012], we use the CUDA code provided by the authors. We use their suggested user parameters (i.e., $7 \times 7$ patch size, and damping factor $\mathrm{k}=0.45$ ) for all the scenes. According to their paper, NLM is tested with a $41 \times 41$ filtering window size for the conference scene, to more aggressively smooth away outliers, while using a $21 \times 21$ filtering window size for the other scenes; note that our method uses the same window size $(19 \times 19)$ across all the scenes to demonstrate the robustness of our method.

We have implemented cross bilateral filtering based on Stein's unbiased risk estimator (SURE) as described in Li et al. [2012]. This method is not limited to a specific filtering method, but the authors recommended the cross bilateral filtering, because it shows the best balance between performance and quality. We have communicated with the authors and used the suggested standard deviation parameters (e.g., 0.8 for normal, 0.25 for texture, and 0.6 for the normalized depth). As suggested by the authors, we prefilter their estimated MSE image using the cross bilateral filtering, and the prefiltered values are used for both their bandwidth selection and sampling map. These parameters are used for all the scenes.

For the block-matching and 3D filtering (BM3D) based adaptive rendering [Kalantari and Sen 2013], we use the code provided by the authors. For the BM3D, we set the smoothing parameter as 3 , as recommended in the paper. 


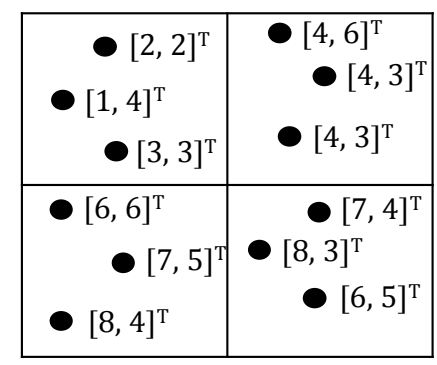

(a) Monte Carlo samples

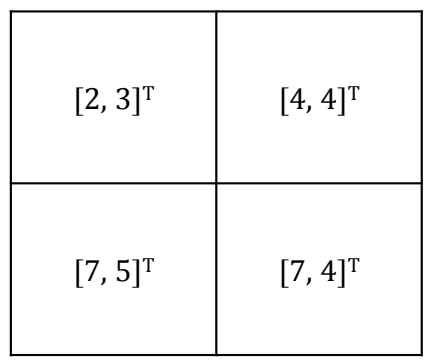

(b) Sample mean $\mathbf{x}^{\mathrm{i}}$

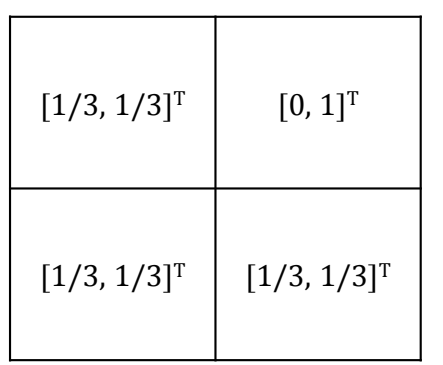

(c) Variance of the sample mean (b)

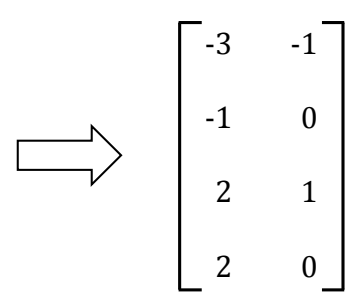

(d) Matrix Z computed from (b)

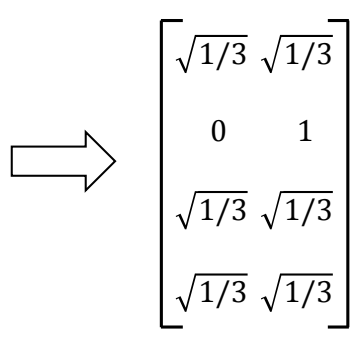

(e) Matrix E using (c)

Fig. 15. This figure shows an example of computing a perturbation matrix $Z=\mu(Z)+E$, where $\mu(Z)$ is the unknown ground truth matrix and $E$ is the error or noise matrix that indicates the perturbation structure introduced by Monte Carlo ray tracing. As shown in (a), we have three samples (i.e., three 2D feature vectors) at each pixel (indicated by a small square). Note that the pixels used for this process are locally defined by a filtering window. In this case, we use a $2 \times 2$ window for simplicity. Given the input samples provided by Monte Carlo ray tracing, we compute the sample mean $\mathbf{x}^{\mathrm{i}} \in \mathbb{R}^{2}(\mathrm{~b})$ and its variance $\operatorname{var}\left(\mathbf{x}^{\mathrm{i}}\right)$ (c) at each pixel. The perturbation matrix $\mathrm{Z}$ (d) is constructed from the sample mean, and whose $i$-th row is set as $\left[\left(\mathbf{x}^{\mathrm{i}}-\overline{\mathbf{x}}\right)^{\mathrm{T}}\right]$, where $\overline{\mathbf{x}}=(5,4)$ is the average of $\mathbf{x}^{\mathrm{i}}$. We calculate each element $E_{i j}$ of the matrix $E$ (e) as $E_{i j}=\left(\operatorname{var}\left(\mathbf{x}^{\mathrm{i}}\right)\right)^{0.5}$. The singular value decomposition on $Z$ leads to $Z=U S V^{T}$, and its non-zero singular values are 4.41 and 0.75 . In order to compute a reduced feature space, the matrix $Z$ is approximated as $Z \approx U_{k} S_{k} V_{k}^{T}$ using $k$ non-zero singular vectors. In this example, the appropriate rank $k$ is estimated as one since 0.75 is lower than our threshold value $\tau=C\|E\|_{2}=2 \times 1.62$, where $\mathrm{C}=2$ is an user parameter that controls truncation effects. As a result, each feature vector $\mathbf{x}^{\mathrm{i}} \in \mathbb{R}^{2}$ defined in a global space is transformed into $\mathbf{z}^{\mathrm{i}}=V_{k}^{T} \mathbf{x}^{\mathrm{i}} \in \mathbb{R}^{1}$. 